\title{
Diagnóstico diferencial das entesopatias Parte 2 - Entesopatias de causas inflamatórias
}

\author{
Michel Alexandre Yazbek ${ }^{1}$, Sônia Maria A. Anti Loduca Lima ${ }^{2}$ \\ DOI: https://doi.org/10.46833/reumatologiasp.2020.19.4.35-38
}

\begin{abstract}
Resumo Algumas doenças inflamatórias imunomediadas podem se manifestar clinicamente como entesites em locais e frequências variadas. As espondiloartrites representam o grupo principal de doenças que se manifestam com entesites. Estudos associaram a presença de entesite com maior atividade de doença, incapacidade funcional e pior qualidade de vida. Em certos casos, a avaliação clínica das entesites é difícil, o que as torna frequentemente subdiagnosticadas. Os métodos de imagem (como a ultrassonografia e a ressonância magnética) podem auxiliar no diagnóstico diferencial entre as entesopatias inflamatórias, mecânicas e a fibromialgia.
\end{abstract}

\section{Unitermos Entesopatia. Espondiloartrite. Espondilite anquilosante. Artrite psoriásica. Artrite juvenil.}

\section{INTRODUÇÃO}

As entesopatias são tradicionalmente consideradas um distúrbio de inserção focal de tendões, ligamentos, cápsulas e fáscias. Apesar desse conceito, os métodos de imagens avançadas e os achados patológicos demonstraram ocorrer um processo inflamatório mais difuso, que também afeta estruturas adjacentes como o osso, tecidos moles e sinóvia ${ }^{1}$.

Algumas doenças reumáticas imunomediadas podem apresentar um processo inflamatório, que se manifestam clinicamente com entesites em locais e frequências variadas ${ }^{1}$. Neste artigo, discutimos as principais doenças inflamatórias envolvidas nesse processo.

\section{DIAGNÓSTICOS DIFERENCIAIS}

\section{Espondiloartrites}

As espondiloartrites (EpAs) representam o grupo principal de doenças inflamatórias que se manifestam com entesite e cons- tituem um grupo heterogêneo de entidades clínicas com fenótipos característicos, que incluem: espondilite anquilosante (EA); artrite psoriásica (APs); artrite associada a doença inflamatória intestinal ou enteropática (AE); artrite reativa (ARe), EpA indiferenciada e EpA juvenil ${ }^{1,2}$. Embora os critérios de Amor $^{3}$, do European Spondylarthropathy Study Group (ESSG) ${ }^{4}$ e do Assessment of Spondyloarthritis International Society (ASAS) ${ }^{5}$ para EpA incluam entesite periférica, há limitações para esses critérios no que diz respeito à quantificação exata de entesite.

As entesites representam um papel-chave na fisiopatogenia das EpAs. Alguns estudos com camundongos transgênicos com TNF (tumour necrosis factor, do inglês), implicaram-na no processo patogênico primário, no qual a lesão mais precoce parece ser na êntese, sendo proposto um "modelo baseado em entesite” como base anatômica unificadora para a osteíte, periostite e neoformação óssea nas EpAs ${ }^{6}$.

As entesites periféricas são observadas em todas as formas de EpAs e os pontos mais comumente detectados são localizados nos membros inferiores, particularmente nos tornozelos,
1. Médico assistente. Doutor da Disciplina de Reumatologia da Universidade Estadual de Campinas (Unicamp), SP. Doutorado pela Unicamp.

2. Médica assistente da Disciplina de Reumatologia da Faculdade de Medicina do ABC (FMABC), Santo André-SP. Preceptora chefe do Ambulatório de Espondiloartrites do Hospital do Servidor Público Estadual de São Paulo (HSPE), SP.

Correspondência: Dr. Michel Alexandre Yazbek, e-mail:m-yazbek@uol.com.br.
Como citar este artigo: Yazbek MA, Lima SMAAL. Diagnóstico diferencial das entesopatias. Parte 2 - Entesopatias de causas inflamatórias. Rev Paul Reumatol. 2020 out-dez;19(4):35-8. DOI: https://doi.org/10.46833/ reumatologiasp.2020.19.4.35-38.

Os autores não contaram com apoio financeiro.

Os autores declaram não ter interesses associativos, comerciais, de propriedade ou financeiros que representem conflito de interesse nos produtos e empresas descritos neste artigo. 
nas inserções dos tendões calcâneos e nas fáscias plantares. Outros pontos frequentes de entesite detectados nos exames ultrassonográficos são: trocânteres femorais, inserção do tendão quadríceps no polo patelar superior e inserções dos tendões flexores e extensores nas falanges ${ }^{7,8,9}$.É importante lembrar que alguns sítios de entesite são clinicamente indetectáveis, incluindo aqueles na coluna e certas articulações profundas, o que contribui para falha de seu diagnóstico nas EpAs.

Palominos et al. correlacionaram os índices Maastricht Ankylosing Spondylitis Enthesitis Score (MASES), Spondyloarthritis Research Consortium of Canada index (SPARCC) e Leeds Enthesitis Index (LEI) com a atividade e a função em pacientes brasileiros com EpAs. Nesse estudo, o índice MASES correlacionou-se um pouco melhor com a atividade de doença e função do que o SPARCC e o LEI. Entretanto, as três pontuações de entesite tiveram apenas uma capacidade razoável para discriminar pacientes com doença em atividade daqueles em remissã $0^{10}$.

As entesites são mais frequentes entre pacientes do sexo feminino versus sexo masculino ${ }^{11-14}$. As prevalências reportadas variam entre $34-74 \%$ e $35 \%$ em pacientes com EA e EpA não radiográfica, respectivamente ${ }^{15-17}$. Uma publicação reportou maior prevalência de manifestações periféricas (entesite e artrite periférica) em pacientes da América Latina comparados com pacientes de países europeus (Bélgica e Espanha) ${ }^{18}$. No Registro Brasileiro de Espondiloartrites (RBE), Carneiro et al. mostraram que a prevalência de entesites é alta ( $54 \%$ entre 1.505 pacientes) e os pontos mais afetados foram a espinha ilíaca posterior e o tendão de calcâneo. Nesse estudo, a entesite foi detectada em $53,5 \%$ dos pacientes com EA, 53,8\% dos pacientes com APs e 70,4\% dos pacientes com EpA indiferenciada ${ }^{19}$.

Alguns estudos demonstraram que a presença de entesite foi associada à maior atividade de doença, incapacidade funcional e para o trabalho, além de pior qualidade de vida em compara- ção com os pacientes sem entesite ${ }^{10-12,16}$. Em pacientes brasileiros, a entesite foi associada a uma maior gravidade das manifestações axiais e periféricas e um valor mais elevado do índice de atividade BASDAI (Bath Ankylosing Disease Activity Index) ${ }^{19,20}$.

A avaliação clínica das entesites é desafiadora, o que as torna frequentemente subdiagnosticadas. A quantificação na prática diária é difícil e carece de sensibilidade e especificidade. A detecção dos pontos depende da dor provocada pela pressão local dos pontos entésicos, sendo que as localizações intra-articulares e profundas de certas ênteses também tornam o exame físico duvidoso e de difícil interpretação ${ }^{21-23}$.

A correlação clínica dos pontos dolorosos nas ênteses no exame físico com os métodos de imagens, como o ultrassom (US) e a ressonância magnética (RM), para detecção de inflamação entésica é baixa ${ }^{24}$. Entretanto, o US pode auxiliar no diagnóstico diferencial entre EpAs e artrite reumatoide (AR) ou fibromialgia (Figura 1). Nos pacientes com EpAs, as entesites ocorrem predominantemente em estruturas extra-articulares, diferentemente dos pacientes com AR, em que a inflamação intra-articular com sinovite é a lesão mais característica ${ }^{25}$. Alguns casos também podem ser confundidos com o fenômeno de sensibilização central da dor, o que ocorre nos pacientes com fibromialgia e síndrome miofascial, por exemplo, e esses métodos de imagem podem auxiliar nesse diagnóstico diferencial ${ }^{26}$.

\section{Artrite psoriásica}

Os estudos em APs associaram a presença de entesite a um pior dano periférico e axial. Além disso, os pacientes com APs e entesite apresentaram maiores índices de atividade de doença e dor, pior qualidade de vida, pior incapacidade funcional, distúrbios de sono e fadiga ${ }^{27-29}$. Alguns investigadores também relataram piores desfechos de doença e uma probabilidade menor de atingir a remissão e a baixa atividade de doença nesses pacientes ${ }^{27,30}$.

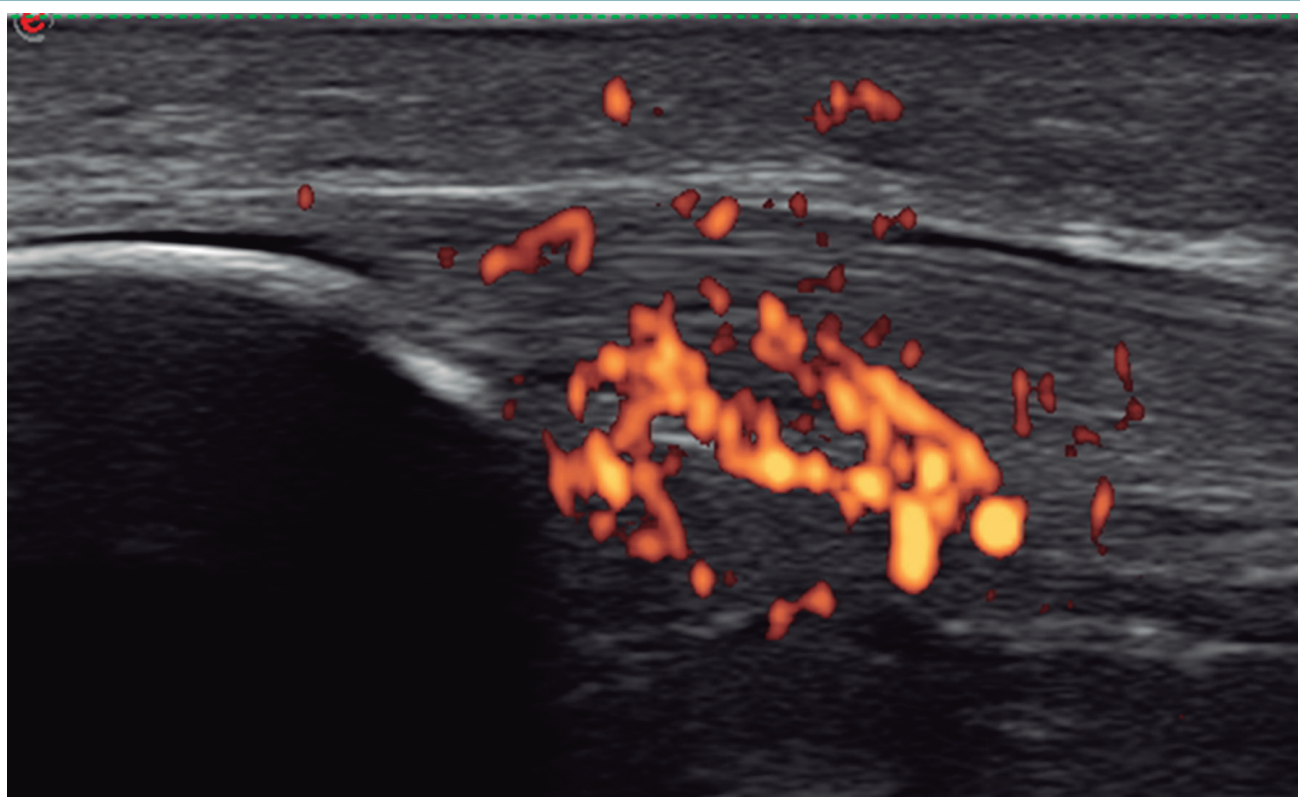

FIGURA 1 Entesite na inserção proximal do tendão patelar em paciente com espondiloartrite. O power Doppler intensamente positivo sugere entesopatia de causa inflamatória. 
A entesite é rotineiramente avaliada em pacientes com APs no registro da Universidade de Toronto pelo índice SPARCC modificado e sua prevalência foi de $35 \%{ }^{28}$. Já o registro norte-americano CORRONA avaliou entesite, através dos índices LEI e SPARCC, encontrando uma prevalência de $27 \%{ }^{27}$.

A presença do diagnóstico de fibromialgia concomitante ocorre em cerca de $20 \%$ dos pacientes com APs e pode resultar em uma piora artefatual da avaliação da gravidade da doença, impedindo o alcance da meta de remissão ou baixa atividade de doença. É preciso se atentar de que as medidas utilizadas no exame físico em estudos e na prática clínica avaliam a sensibilidade relatada do paciente, que pode representar tanto um local verdadeiro de entesite, como também refletir o fenômeno de dor generalizada em pacientes com fibromialgia ou sensibilização central da dor. 0 fato de que uma inflamação pode não estar sempre presente em uma área sensível é confirmado, em alguns estudos, pela falta de correlação com a evidência de inflamação por US ou $\mathrm{RM}^{27}$.

\section{Doença inflamatória intestinal}

A entesopatia inflamatória ainda é pouco estudada em pacientes com doença inflamatória intestinal, sendo encontrada em frequências muito variadas na literatura internacional, entre 7\% e $50 \%$ dos pacientes com doença inflamatória intestinal (DII) ${ }^{31}$.

Em um estudo brasileiro, Lanna et al. avaliaram 130 pacientes com DII. Dentre esses pacientes, sete (5,4\%) apresentaram entesite, sendo a inserção do tendão de calcâneo a êntese mais frequentemente envolvida (seis pacientes) ${ }^{32}$. Resende et $a l$. avaliaram a casuística do RBE e encontraram uma frequência de 3,2\% de AE entre os pacientes com EpAs, sendo 2,5\% com espondilite e $0,7 \%$ com artrite. Esse subgrupo de indivíduos com AE teve maior prevalência em mulheres e uma menor incidência de entesite, comparado com os outros pacientes com $\mathrm{EpAs}^{33}$.

\section{Artrite relacionada com entesite}

A entesite periférica é observada com frequência na EpA de início juvenil, particularmente naqueles classificados como portadores de artrite relacionada com entesite (ERA). Dentre os subtipos de artrite idiopática juvenil (AIJ), a ERA é aquele que está associado a piores função e qualidade de vida ${ }^{34,35}$.
A ERA é mais comum em crianças do sexo masculino, com idade acima de 6 anos e está associada majoritariamente à presença do antígeno de histocompatibilidade humano B27 (do inglês, histocompatibility leukocyte antigen B27 ou HLA-B27). Os pacientes geralmente apresentam quadro clínico de entesite, oligoartrite assimétrica, predominantemente em membros inferiores, podendo estar associada ou não à sacroiliíte ${ }^{36}$.

A entesite é uma manifestação clínica característica da ERA, podendo ser a manifestação inicial, estando presente em mais da metade dos pacientes. As ênteses mais comumente afetadas são: fáscia plantar, tendão calcâneo, porções superior e inferior da patela, tuberosidade tibial, trocânter maior do fêmur, espinha ilíaca, crista ilíaca, junção costocondral, locais de inserção do manguito rotador, epicôndilos medial e lateral do cotovelo e processos espinhosos dos segmentos cervical, torácico e lombar da coluna ${ }^{37}$.

De acordo com os critérios da International League of Associations for Rheumatology (ILAR), a classificação de ERA requer a presença de artrite com entesite ou a presença de artrite ou entesite associada com qualquer uma das seguintes características: uveíte sintomática, início de artrite em menino com seis anos de idade ou mais, positividade do HLA-B27, dor lombar inflamatória ou dor à palpação das articulações sacrilíacas, ou história familiar de uma doença associada ao HLA-B27, como EpA (adulta ou juvenil), ARe, AE ou uveíte anterior aguda em um parente de primeiro grau ${ }^{36}$.

\section{CONCLUSÃO}

O diagnóstico diferencial das entesites continua sendo um desafio para o reumatologista em sua prática clínica. Dentre as causas inflamatórias, as espondiloartrites em geral destacam-se como o grupo principal de doenças que se manifestam com entesite. Vários estudos demonstraram um impacto da entesite na piora da atividade de doença, da função e da qualidade de vida.

Há situações clínicas em que a diferenciação com entesopatias mecânicas, com outras doenças inflamatórias ou com o fenômeno da sensibilização central (como a fibromialgia) é difícil. Nesses casos, a aquisição de imagem (US ou RM) é um importante recurso diagnóstico, pois pode mostrar características que sugerem a causa da entesite.

\section{REFERÊNCIAS}

1. Kehl AS, Corr M, Weisman MH. Review: Enthesitis: New Insights Into Pathogenesis, Diagnostic Modalities, and Treatment. Arthritis Rheumatol. 2016;68(2):312-22. PMID: 26473401; PMCID: PMC5195265. https://doi. org/10.1002/art.39458.

2. Sieper J, Rudwaleit M, Baraliakos X, Brandt J, Braun J, Burgos-Vargas R, et al. The Assessment of SpondyloArthritis international Society (ASAS) handbook: a guide to assess spondyloarthritis. Ann Rheum Dis. 2009;68 Suppl 2:ii1-44. https://doi.org/10.1136/ard.2008.104018.

3. Amor B, Dougados M, Mijiyawa M. Criteria for the classification of spondylarthropathies. Rev Rhum Mal Osteoartic. 1990;57:85-9. In French. [PubMed: 2181618]

4. Dougados M, van der Linden S, Juhlin R, Huitfeldt B, Amor B, Calin A, et al. the European Spondylarthropathy Study Group. The European Spondylarthropathy Study Group preliminary criteria for the classification of spon- dylarthropathy. Arthritis Rheum. 1991;34:1218-27. https://doi.org/10.1002/ art.1780341003.

5. Rudwaleit M, Landewé R, van der Heijde D, Listing J, Brandt J, Braun J, et al. The development of Assessment of SpondyloArthritis international Society classifi cation criteria for axial spondyloarthritis (part I): classification of paper patients by expert opinion including uncertainty appraisal. Ann Rheum Dis. 2009;68:770-6. https://doi.org/10.1136/ard.2009.108217.

6. Jacques P, Lambrecht S, Verheugen E, Pauwels E, Kollias G, Armaka M, et al. Proof of concept: enthesitis and new bone formation in spondyloarthritis are driven by mechanical strain and stromal cells. Ann Rheum Dis. 2014;73:437-45. [PubMed: 23921997] https://doi.org/10.1136/annrheumdis-2013-203643.

7. D'Agostino MA, Aegerter P, Bechara K, Salliot C, Judet O, Chimenti MS, et al. How to diagnose spondyloarthritis early? Accuracy of peripheral enthesitis detection by power Doppler ultrasonography. Ann Rheum Dis. 2011;70:143340. https://doi.org/10.1136/ard.2010.138701. 
8. Weill C, Norregaard J, Szkudlarek M, Hasselquist M, Moller JM, Terslev L, et al. Ultrasonography of finger joints, tendons and entheses in patients with spondyloarthropathy: a comparison with clinical examination and MRI. Ann Rheum Dis. 2005;64:327.

9. D’Agostino MA, Olivieri I. Enthesitis. Best Pract Res Clin Rheumatol. 2006;20:473-86. https://doi.org/10.1016/j.berh.2006.03.007.

10. Palominos PE, de Campos AP, Ribeiro SL, Xavier RM, Xavier JW, de Oliveira FB, et al. Correlation of enthesitis indices with disease activity and function in axial and peripheral spondyloarthritis: a cross-sectional study comparing MASES, SPARCC and LEI. Adv Rheumatol. 2019;59:23. https://doi. org/10.1186/s42358-019-0066-8.

11. Mease PJ, Liu M, Rebello S, Hua W, McLean RR, Yi E, et al. Characterization of patients with axial spondyloarthritis by enthesitis presence: data from the Corrona Psoriatic Arthritis/Spondyloarthritis Registry. ACR Open Rheumatol. 2020;2(7):449-56. https://doi.org/10.1002/acr2.11154.

12. Tournadre A, Pereira B, Lhoste A, Dubost JJ, Ristori JM, Claudepierre P, et al. Differences between women and men with recent-onset axial spondyloarthritis: results from a prospective multicenter French cohort. Arthritis Care Res. (Hoboken) 2013;65:1482-9. https://doi.org/10.1002/acr.22001.

13. Lubrano E, Perrotta FM, Manara M, D’Angelo S, Addimanda O, Ramonda R, et al. The sex influence on response to tumor necrosis factor- $\alpha$ inhibitors and remission in axial spondyloarthritis. J Rheumatol. 2018;45:195-201. https:// doi.org/10.3899/jrheum.17666.

14. Shahlaee A, Mahmoudi M, Nicknam MH, Farhadi E, Fallahi S, Jamshidi AR. Gender differences in Iranian patients with ankylosing spondylitis. Clin Rheumatol. 2015;34:285-93. https://doi.org/10.1007/s10067-013-2439-4.

15. Ciurea A, Scherer A, Exer P, Bernhard J, Dudler J, Beyeler B, et al. Tumor necrosis factor $\alpha$ inhibition in radiographic and nonradiographic axial spondyloarthritis: results from a large observational cohort. Arthritis Rheum. 2013;65:3096-106. https://doi.org/10.1002/art.38140.

16. De Winter JJ, van Mens LJ, van der Heijde D, Landewé R, Baeten DL. Prevalence of peripheral and extra-articular disease in ankylosing spondylitis versus non-radiographic axial spondyloarthritis: a metaanalysis. Arthritis Res Ther. 2016;18:196. https://doi.org/10.1186/s13075-016-1093-Z.

17. Vander Cruyssen B, Ribbens C, Boonen A, Mielants H, de Vlam K, Lenaerts J, et al. The epidemiology of ankylosing spondylitis and the commencement of anti-TNF therapy in daily rheumatology practice. Ann Rheum Dis. 2007;66:1072-7. https://doi.org/10.1136/ard.2006.064543.

18. Benegas M, Munoz-Gomariz E, Font P, Burgos-Vargas R, Chaves J, Palleiro D, et al. Comparison of the clinical expression. Of patients with ankylosing spondylitis from Europe and Latin America. J Rheumatol. 2012; 39:2315-20. https://doi.org/10.3899/jrheum.110687.

19. Carneiro S, Bortoluzzo A, Gonçalves C, da Silva JAB, Ximenes AC, Bertolo M, et al. Effect of enthesitis on 1505 Brazilian patients with Spondyloarthritis. J Rheumatol. 2013;40:1719-25. https://doi.org/10.3899/jrheum.121145.

20. Da Costa IP, Bortoluzzo AB, Gonçalves AR, da Silva JAB, Ximenes AC, Bértolo $M B$, et al. Evaluation of performance of BASDAI (Bath ankylosing spondylitis disease activity index) in a Brazilian cohort of 1492 patients with spondyloarthritis: data from the Brazilian registry of Spondyloarthritides (RBE). Rev Bras Reumatol. 2015;55:48-54. https://doi.org/10.1016/j.rbre.2014.05.005.

21. D'Agostino MA, Said-Nahal R, Hacquard-Bouder C, Brasseur JL, Dougados M, Breban M. Assessment of peripheral enthesitis in the spondylarthropathies by ultrasonography combined with power Doppler: a cross-sectional study. Arthritis Rheum. 2003;48:523-33. [PubMed: 12571863] https://doi. org/10.1002/art.10812.

22. Wiell C, Norregaard J, Szkudlarek M, Hasselquist M, Moller JM, Terslev L, et al. Ultrasonography of finger joints, tendons and entheses in patients with spondyloarthropathy: a comparison with clinical examination and MRI. Ann Rheum Dis. 2005;64(Suppl III):327.

23. Wiell C, Norregaard J, Szkudlarek M, Hasselquist M, Moller JM, Terslev L, et al. Ultrasonography of lower extremity tendons and entheses in patients with spondyloarthropathy: a comparison with clinical examination and MRI. Ann Rheum Dis. 2005;64(Suppl III):378.

24. Zhang H, Liang J, Qiu J, Wang F, Sun L. Ultrasonographic evaluation of enthesitis in patients with ankylosing spondylitis. J Biomed Res 2017;31:162-9.

25. Godfrin B, Zabraniecki L, Lamboley V, Bertrand-Latour F, Sans N, Fournié B. Spondyloarthropathy with entheseal pain. A prospective study in 33 patients. Joint Bone Spine 2004;71:557-62. https://doi.org/10.1016/j.jbspin.2003.10.010.

26. Mease PJ. Fibromyalgia, a missed comorbidity in spondyloarthritis: prevalence and impact on assessment and treatment. Curr Opin Rheumatol. 2017;29:304-10. https://doi.org/10.1097/BOR.0000000000000388.

27. Mease PJ, Karki C, Palmer JB, Etzel CJ, Kavanaugh A, Ritchlin CT, et al. Clinical characteristics, disease activity, and patient-reported outcomes in psoriatic arthritis patients with dactylitis or enthesitis: results from the Corrona Psoriatic Arthritis/Spondyloarthritis Registry. Arthritis Care Res. (Hoboken) 2017;69:1692-9. https://doi.org/10.1002/acr.23249.

28. Polachek A, Li S, Chandran V, Gladman DD. Clinical enthesitis in a prospective longitudinal psoriatic arthritis cohort: incidence, prevalence, characteristics, and outcome [published erratum appears in Arthritis Care Res (Hoboken) 2019; 71:574]. Arthritis Care Res. (Hoboken) 2017;69:1685-91. https://doi. org/10.1002/acr.23174.

29. Polachek A, Cook R, Chandran V, Gladman DD, Eder L. The association between sonographic enthesitis and radiographic damage in psoriatic arthritis. Arthritis Res Ther. 2017;19:189. https://doi.org/10.1186/s13075-0171399-5.

30. Kaeley GS, Eder L, Aydin SZ, Gutierrez M, Bakewell C. Enthesitis: a hallmark of psoriatic arthritis. Semin Arthritis Rheum 2018;48:35-43. https://doi. org/10.1016/j.semarthrit.2017.12.008.

31. Salvarani C, Fries W. Clinical features and epidemiology of spondyloarthritides associated with inflammatory bowel disease. World J Gastroenterol. 2009;15(20):2449-55. https://doi.org/10.3748/wjg.15.2449.

32. Lanna CC, Ferrari MLA, Rocha SL, Nascimento E, de Carvalho MA, da Cunha AS. A cross-sectional study of 130 Brazilian patients with Crohn's disease and ulcerative colitis: analysis of articular and ophthalmologic manifestations. Clin Rheumatol. 2008;27(4):503-9. Epub 2007 Dec 21. PMID: 18097711. https://doi.org/10.1007/s10067-007-0797-5.

33. Resende GG, Lanna CCD, Bortoluzzo AB, Gonçalves CR, Silva JAB, Ximenes AC et al. Artrite enteropática no Brasil: dados do registro brasileiro de espondiloartrites. Rev Bras Reumatol. 2013;53(6):452-9. https://doi.org/10.1016/j. rbr.2013.04.001.

34. Weiss P, Beukelman T, Schanberg LE, Kimura Y, Colbert RA. CARRAnet Investigators. Enthesitis is a significant predictor of decreased quality of life, function, and arthritis-specific pain across juvenile idiopathic arthritis (JIA) categories: preliminary analyses from the CARRAnet registry. Arthritis Rheum. 2011;63(Suppl):S105.

35. Weiss PF. Evaluation and treatment of enthesitis-related arthritis. Curr Med Lit Rheumatol. 2013;32:33-41. [PubMed: 24403667]

36. Mistry RR, Patro P, Agarwal V, Misra DP. Enthesitis-related arthritis: current perspectives. Open Access Rheumatol. 2019;11:19-31. https://doi. org/10.2147/OARRR.S163677.

37. Ramanathan A, Srinivasalu H, Colbert RA. Update on juvenile spondyloarthritis. Rheum Dis Clin North Am. 2013;39(4):767-88. https://doi.org/10.1016/j. rdc.2013.06.002 